Pontifícia Universidade Católica $_{\text {a }}$ DO RIO DE JANEIRO

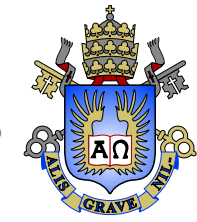

Frederico Carvalho Gomes

\title{
Modelo Assintótico do Deslocamento de Líquidos em Anulares com Excentricidade \\ Variável
}

Dissertação de Mestrado

Dissertação apresentada como requisito parcial para obtenção do grau de Mestre pelo Programa de Pós-graduação em Engenharia Mecânica do Departamento de Engenharia Mecânica da PUCRio

Orientador: Prof. Márcio da Silveira Carvalho 
Frederico Carvalho Gomes

\author{
Modelo Assintótico do Deslocamento de \\ Líquidos em Anulares com Excentricidade \\ Variável
}

Dissertação apresentada como requisito parcial para obtenção do grau de Mestre pelo Programa de Pós-graduação em Engenharia Mecânica do Departamento de Engenharia Mecânica do Centro Técnico Científico da PUC-Rio. Aprovada pela Comissão Examinadora abaixo assinada.

\author{
Prof. Márcio da Silveira Carvalho \\ Orientador \\ Departamento de Engenharia Mecânica - PUC-Rio
}

Prof. Luis Fernando Alzuguir Azevedo

Departamento de Engenharia Mecânica - PUC-Rio

Prof. Andre Leibsohn Martins

CENPES - Petrobras

Prof. José Eugênio Leal

Coordenador Setorial do Centro Técnico Científico - PUC-Rio 
Todos os direitos reservados. É proibida a reprodução total ou parcial do trabalho sem autorização da universidade, do autor e do orientador.

\section{Frederico Carvalho Gomes}

Frederico Carvalho Gomes graduou-se em Engenharia Mecânica e de Produção Mecânica pela Pontifícia Universidade Católica do Rio de Janeiro [2005].

Ficha Catalográfica

Gomes, Frederico Carvalho

Modelo Assintótico do Deslocamento de Líquidos em Anulares com Excentricidade Variável / Frederico Carvalho Gomes; orientador: Márcio da Silveira Carvalho. - Rio de Janeiro : PUC-Rio, Departamento de Engenharia Mecânica, 2009.

v., 54 f: il. ; $29,7 \mathrm{~cm}$

1. Dissertação (mestrado) - Pontifícia Universidade Católica do Rio de Janeiro, Departamento de Engenharia Mecânica.

Inclui referências bibliográficas.

1. Engenharia Mecânica - Tese. 2. Escoamento em Anular. 3. Teoria da Lubrificação. 4. Processo de Cimentação. I. Carvalho, Márcio da Silveira. II. Pontifícia Universidade Católica do Rio de Janeiro. Departamento de Engenharia Mecânica. III. Título. 


\section{Agradecimentos}

À minha família por tudo.

Ao meu orientador Professor Márcio Carvalho pelo apoio e incentivo para a realização deste trabalho.

Aos meus amigos e colegas da PUC-Rio e do TecGraf.

Ao CNPq, CAPES, FAPERJ e à PUC-Rio, pelos auxílios concedidos. 


\section{Resumo}

Gomes, Frederico Carvalho; Carvalho, Márcio da Silveira. Modelo Assintótico do Deslocamento de Líquidos em Anulares com Excentricidade Variável. Rio de Janeiro, 2009. 54p. Dissertação de Mestrado - Departamento de Engenharia Mecânica, Pontifícia Universidade Católica do Rio de Janeiro.

Uma das etapas do processo de perfuração de um poço de petróleo é a cimentação, ela prove o isolamento das zonas e a sustentação do revestimento. Durante o processo de cimentação é necessário a substituição da lama de perfuração pela pasta de cimento. Para evitar a mistura entre esses fluidos é normalmente utilizado um fluido espaçador. Dessa forma é comum que sejam bombeados 3 ou mais fluidos através do anular excêntrico formado pela parede da rocha e o revestimento. A análise completa do escoamento no espaço anular que ocorre durante o processo de cimentação é extremamente complexa, pois os fluidos em questão podem apresentar comportamento não-Newtoniano e o escoamento é tridimensional e em regime transiente. Desta forma, a análise computacional do processo é extremamente cara. Modelos simplificados para o processo estão disponíveis na literatura e são largamente utilizados em softwares comerciais pela industria de exploração de petróleo. No entanto, as muitas simplificações adotadas nestes modelos limitam os parâmetros nos quais são obtidos bons resultados. Neste trabalho, foi desenvolvido um modelo 2D para esse escoamento 3D utilizando as simplificações propostas pela teoria de lubrificação. O modelo desenvolvido considera a variação de inclinação e excentricidade ao longo do poço e as características não-Newtonianas dos fluidos envolvidos. Os resultados obtidos mostram como as propriedades dos fluidos e a geometria do poço afetam a eficiência do processo de bombeio.

\section{Palavras-chave}

Escoamento em Anular; Teoria da Lubrificação; Processo de Cimentação; 


\section{Abstract}

Gomes, Frederico Carvalho; Carvalho, Márcio da Silveira (Adviser). Asymptotic Model for Displacement of Liquids in Annular with Variable Eccentric. Rio de Janeiro, 2009. 54p. MSc Thesis - Departamento de Engenharia Mecânica, Pontifícia Universidade Católica do Rio de Janeiro.

The cementation process is an important step in the construction of oil and gas wells. It provides zonal isolation and support for the well bore. During the cementation, it is necessary to displace the drilling mud by the cement slurry. To avoid mixing of these liquids, spacer fluids are usually used. Therefore, it is common to have three or more liquids flowing through the eccentric annular space. A complete analysis of the flow in the annular space that occurs during cementation is extreme complex, because of the presence of different liquids, that often present non-Newtonian characteristic and the flow is three dimensional and transient. Thus, a complete model has a prohibitive high computational cost. Simplified models are available in the literature and are used by the oil industry in commercial simulation software for cementation. However, the strong simplifying assumptions limit the range of parameters at which these models are accurate. In this work, a 2D model for this 3D flow was developed using the lubrication theory. The developed model considered the variation of the inclination and eccentricity along the well and the non-Newtonian behavior of the flowing liquids. The results show how the liquid properties and well geometry affect the efficiency of the displacement process.

\section{Keywords}

Annular Flow; Lubrication Theory; Cementation Process; 


\section{Sumário}

1 Introdução $\quad 10$

2 Formulação do Problema $\quad 15$

2.1 Considerações Gerais $\quad 15$

2.2 Equação da Quantidade de Movimento 16

$\begin{array}{ll}2.3 \text { Teoria de Lubrificação } & 18\end{array}$

2.4 Equação de Continuidade 20

2.5 Discretização do modelo 22

2.6 Avanço da Interface 25

2.7 Modelo Não-Newtoniano 29

3 Simulação Numérica e Resultados 32

3.1 Avaliação da Precisão do Modelo em Coordenadas Cartesianas 33

3.2 Exemplo Poço vertical - Newtoniano - 2 Fluidos 36

3.3 Exemplo Poço vertical - não-Newtoniano - 3 Fluidos 38

3.4 Exemplo Poço Horizontal 40

3.5 Exemplo Excentricidade Variável 42

3.6 Exemplo Geometrial Real 46

4 Considerações Finais e Trabalhos Futuros $\quad 51$

$\begin{array}{ll}\text { Referências Bibliográficas } & 53\end{array}$ 


\section{Lista de figuras}

1.1 Exemplo esquemático de um poço 10

1.2 Exemplo esquemático da perfuração do poço 11

2.1 Geometria do problema - vista lateral $\quad 15$

$\begin{array}{ll}2.2 \text { Geometria do problema - vista superior } & 16\end{array}$

2.3 Domínio do modelo 23

2.4 Discretização do modelo 23

2.5 Detalhe da discretização do modelo 24

2.6 Função degrau 28

2.7 Função degrau 28

2.8 Fluxograma de solução para fluido não-Newtoniano 30

3.1 Fluxograma de solução 32

3.2 Campo de pressão - Caso 5

3.3 Gráfico de avanço da interface - Exemplo Vertical Newtoniano 37

3.4 Geometria do exemplo - Vertical não-Newtoniano 38

3.5 Gráfico de avanço da interface - Exemplo Vertical não-Newtoniano 39

3.6 Gráfico de avanço da interface - Exemplo Poço Horizontal 42

3.7 Geometria do exemplo em escala - Exemplo Excentricidade Variável 43

3.8 Gráfico de avanço da interface - Exemplo Excentricidade Variável 44

3.9 Gráfico de avanço da interface - Exemplo Excentricidade Variável 45

3.10 Geometria do exemplo em escala - Exemplo Geometria Real 46

3.11 Gráfico de avanço da interface - Exemplo Geometria Real 48

3.12 Gráfico de avanço da interface - Exemplo Geometria Real 49

3.13 Gráfico de concentração - Exemplo Geometria Real 50

4.1 Iso-linhas de pressão 52 


\section{Lista de tabelas}

3.1 Dados para avalidação do modelo Newtonino 33

3.2 Resultados para avalidação do modelo Newtonino 34

3.3 Dados para avalidação do modelo não-Newtonino 34

3.4 Resultados para avalidação do modelo não-Newtonino 35

3.5 Fluidos bombeados - Exemplo Vertical Newtoniano 36

3.6 Dados da geometria - Exemplo Vertical Newtoniano 36

3.7 Comparação da Posição da Interface - Exemplo Vertical Newtoniano 36

3.8 Dados da geometria - Exemplo Vertical não-Newtoniano 38

3.9 Fluidos bombeados - Exemplo Vertical não-Newtoniano 39

3.10 Dados da geometria - Exemplo Poço Horizontal 40

3.11 Fluidos bombeados - Exemplo Poço Horizontal 41

3.12 Dados dos segmentos - Exemplo Excentricidade Variável 43

3.13 Fluidos bombeados - Exemplo Excentricidade Variável 43

3.14 Dados dos segmentos - Exemplo Geometria Real 46

3.15 Dados dos Fluidos - Exemplo Geometria Real 47 